\title{
B-cell acute lymphoblastic leukemia associated with SET-NUP214 rearrangement: A case report and review of the literature
}

\author{
HONG-HU ZHU, XIAO-SU ZHAO, YA-ZHEN QIN, YUE-YUN LAI and HAO JIANG \\ Department of Hematology, Peking University People's Hospital, Peking University Institute of Hematology, \\ Beijing Key Laboratory of Hematopoietic Stem Cell Transplantation, Beijing 100044, P.R. China
}

Received December 10, 2014; Accepted January 15, 2016

DOI: $10.3892 / \mathrm{ol} .2016 .4260$

\begin{abstract}
The SET nuclear proto-oncogene (SET)-nucleoporin (NUP)214 fusion gene, which results from cryptic $\mathrm{t}(9 ; 9)(\mathrm{q} 34 ; \mathrm{q} 34)$ or $\operatorname{del}(9)(\mathrm{q} 34.11 \mathrm{q} 34.13)$, is a rare genetic event in hematological malignancies. The majority of patients carrying SET-NUP214 experience T-cell acute lymphoblastic leukemia (T-ALL), but rarely experience acute undifferentiated leukemia or acute myeloid leukemia. The current study presents the case of a 19-year-old male patient with B-cell ALL (B-ALL) carrying the SET-NUP214 fusion gene, in addition to an fms-related tyrosine kinase 3-internal tandem duplication mutation and a complex karyotype abnormality. The patient exhibited chemotherapy resistance. To the best of our knowledge, the present study is the first report of a case of B-ALL carrying the SET-NUP214 fusion gene, and provides a review of the literature.
\end{abstract}

\section{Introduction}

Recurrent genetic abnormalities are diagnostic and prognostic markers that enable the classification of acute leukemia into distinct categories and aid the selection of treatment (1-3). Cryptic t(9;9)(q34;q34) and del(9)(q34.11q34.13) are rare genetic abnormalities that lead to the formation of the SET nuclear proto-oncogene (SET)-nucleoporin (NUP)214 fusion gene, which is a marker of acute leukemia (1-12). SET-NUP214 was first detected in a patient with acute undifferentiated leukemia (AUL) (1), and later detected in two patients with acute myeloid leukemia (AML) (2,5). Recent increasing evidence supports an association between SET-NUP214 and pediatric and adult T-cell acute lymphoblastic leukemia (T-ALL) with a frequency of $3.3-10.3 \%$ (3,4,6-12). Gorello et al (4) reported an estimated incidence of SET-NUP214 in adult T-ALL patients of $4.6 \%$ (7/152 cases) (4). The current typical treatment for T-ALL

Correspondence to: Dr Hong-Hu Zhu, Department of Hematology, Peking University People's Hospital, Peking University Institute of Hematology, Beijing Key Laboratory of Hematopoietic Stem Cell Transplantation, 11 Xizhimen South Street, Beijing 100044, P.R. China E-mail: zhuhhdoc@163.com

Key words: acute lymphoblastic leukemia, SET-NUP214, treatment patients with SET-NUP214 is allogeneic hematopoietic stem cell transplantation (allo-HSCT), as chemotherapy resistance is common (12). The 3-year overall survival (OS) rate was reported to be $73 \%$ in 9 patients who received allo-HSCT (12). In addition, compared with SET-NUP214-negative patients, SET-NUP214-positive patients demonstrated a significantly increased rate of corticosteroid and chemotherapy resistance; however, this was not observed to negatively influence clinical outcome following allogeneic transplantation (12). However, there is not sufficient information on the clinical characteristics and treatment outcomes of patients carrying the SET-NUP214 fusion gene (1-12). Furthermore, to the best of our knowledge, no case of B-cell ALL (B-ALL) carrying the SET-NUP214 fusion gene has been reported thus far.

In the present study, the first case of B-ALL carrying the SET-NUP214 fusion gene is reported, and the literature regarding patients with SET-NUP214 is reviewed in order to provide a comprehensive profile of this rearrangement.

\section{Case report}

On August 14, 2014, a 19-year-old man was referred to the Peking University People's Hospital (Beijing, China) with complaints of recurrent fever, fatigue, dizziness and paleness during the previous month. Clinical examination revealed systemic superficial lymph node enlargement, tenderness of the sternum and severe splenomegaly (subcostal, $11 \mathrm{~cm}$ ). Peripheral blood count revealed a white blood cell (WBC) count of $217 \times 10^{9}$ cells $/ 1$, hemoglobin count of $78 \mathrm{~g} / 1$ and platelet count of $40 \times 10^{9}$ cells/1 consisting of $92.5 \%$ blasts. The bone marrow displayed hypercellularity and diffuse infiltration of leukemic lymphoblasts $(93.5 \%)$. Histochemical staining revealed peroxidase negative (100\%) and periodic acid Schiff positive staining (weak staining, 60\%; moderate staining, 15\%; and strong staining, 1\%). Immunophenotypic analysis revealed that the bone marrow cells were human leukocyte antigen-antigen D Related (HLA-DR) ${ }^{+}$, cluster of differentiation (CD) $34^{+}, \mathrm{CD}^{+} 8^{+}$, $\mathrm{CD}^{+}{ }^{+}$, cytoplasmic (c)CD79a ${ }^{+}, \mathrm{CD}_{19}{ }^{+}(\mathrm{dim}), \mathrm{CD} 22^{+}(\mathrm{dim})$,

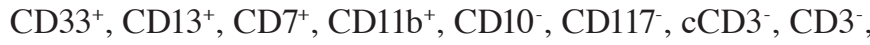

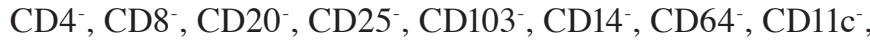
FMC7- $^{-}$, c myeloperoxidase (MPO) ${ }^{-}$, c immunoglobulin (Ig) $\mathrm{M}^{-}, \operatorname{Ig} \kappa^{-}$and $\operatorname{Ig} \lambda$. The antibodies used for the present analysis were all monoclonal mouse anti-human and did not require dilution. The volume of each antibody added was $20 \mu \mathrm{l}$ for 
$5 \times 10^{5}$ cells, and cells were incubated in $1 \%$ bovine serum albumin and phosphate-buffered saline (Sigma-Aldrich, St. Louis, MO, USA) at room temperature for $15 \mathrm{~min}$. The antibodies were directed against the following proteins: CD58 (catalog no. IM1218U), cCD79a (catalog no., IM2221), CD7 (catalog no., A07755), CD4 (catalog no., A07751), CD8 (catalog no., A07756), CD14 (catalog no., IM0645U) and CD64 (catalog no., IM3601U) (all purchased from Beckman Coulter, Inc., Brea, CA, USA); HLA-DR (catalog no., 340549), CD34 (catalog no., 348053), CD38 (catalog no., 345807), CD19 (catalog no., 340437), CD22 (catalog no., 347577), CD33 (catalog no., 340474), CD13 (catalog no., 347837), CD11b (catalog no., 340937), CD117 (catalog no., 340529), CD20 (catalog no., 347673), CD25 (catalog no., 341009), CD11c (catalog no., 340544), FMC7 (catalog no., 340919), MPO (catalog no., 340580), CD10 (catalog no., 555375), cCD3

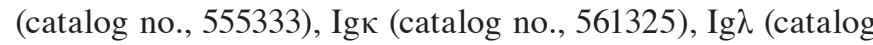
no., 555793), CD103 (catalog no., 550260) and cIgM (catalog no., 562030) (all purchased from BD Biosciences, San Jose, CA, USA). The karyotype of the bone marrow cells was $56, X Y,+6,+8,+12,+13,+15,+19,+20,+21,+21,+\operatorname{mar}(1) / 45-49$ and $48, X Y,+12,+15,+16, \mathrm{i}(17)(\mathrm{q} 10),+21,+22,+\operatorname{mar} 2(\mathrm{cp} 5) / 46, \mathrm{XY}(4)$. Multiplex reverse transcription-polymerase chain reaction (RT-PCR) of the bone marrow cells revealed the presence of a SET-NUP214 fusion transcript of 393 base pairs and an fms-related tyrosine kinase 3-internal tandem duplication (ITD) mutation. Subsequent cloning and sequencing confirmed the fusion of exon 7 of the SET gene and the exon 18 of the NUP214 gene. Therefore, the patient was diagnosed with B-ALL and SET-NUP214 rearrangement. The patient then received induction chemotherapy with cyclophosphamide, vindesine, daunorubicin and prednisone (COPD) regimen (cyclophosphamide, $750 \mathrm{mg} / \mathrm{m}^{2}$, day 1; vindesine, $4 \mathrm{mg} / \mathrm{m}^{2}$, days $1,8,15$ and 22 ; daunorubicin, $40 \mathrm{mg} / \mathrm{m}^{2}$, days $1-3$; prednisone, $1 \mathrm{mg} / \mathrm{kg}$, days $1-28)$. The proportion of lymphoblasts was $96.0 \%$ of blood cells (normal, $0 \%$ ) and $97.5 \%$ of bone marrow cells (normal, $0-0.5 \%$ ) following one week of induction chemotherapy, and 6.0 and $34.0 \%$, respectively, three weeks later. However, the patient did not achieve complete remission, and is currently awaiting for allogeneic hematopoietic stem cell transplantation (allo-HSCT). The present report was conducted in accordance with the guidelines of the Declaration of Helsinki, and informed consent was obtained from the patient.

Clinical characteristics of acute leukemia associated with SET-NUP214 based on a review of the literature. Including the present case, a total of 42 patients with acute leukemia carrying SET-NUP214 have been reported to date (Table I). These patients presented different subtypes of leukemia, including T-ALL (38/42; 90.5\%), AUL (2/42; 4.8\%), AML (1/42; 2.4\%) and B-ALL $(1 / 42 ; 2.4 \%)$. The incidence of SET-NUP214 among patients with T-ALL was observed to be $3.3-10.3 \%$. The median age was 27.5 years (range, 8.0-56.0 years), and $72.5 \%(29 / 40)$ of cases were men. The median WBC count was $30.9 \times 10^{9}$ cells $/ 1$ (range, $1.5-604.4 \times 10^{9}$ cells $/ 1$ ). The median percentage of leukemic blasts in the bone marrow was $91.0 \%$ (range, 82.0-97.0\%). The common immunophenotype was $\mathrm{CD}^{2} 4^{+}(22 / 27 ; 81.5 \%), \mathrm{CD}_{3}{ }^{+}(25 / 29 ; 86.2 \%), \mathrm{cCD}^{+}(25 / 26$; 96.2\%), $\mathrm{CD}^{+}(29 / 29 ; 100.0 \%)$ and $\mathrm{CD}^{+} 3^{+}(9 / 19 ; 47.4 \%)$. Of
31 patients, $38.7 \%(12 / 31)$ carried a normal karyotype, $32.3 \%$ $(10 / 31)$ carried a complex karyotype, $3.3 \%$ (1/31) carried $\mathrm{t}(9 ; 9)(\mathrm{q} 34 ; \mathrm{q} 34)$ and $25.8 \%$ carried other type of karyotype. Interphase and metaphase fluorescence in situ hybridization (FISH) analysis using Vysis LSI BCR/ABL Dual Color Dual Fusion Translocation Probe (Abbott Molecular, Des Plaines, IL, USA) revealed del(9)(q34)/ABL in 20 patients whose results were available. Molecular abnormalities included mutations in NOTCH1 and plant homeodomain-like finger (PHF)6 and overexpression of homeobox A (HOXA) in $91.7 \%(11 / 12), 66.7 \%(4 / 6)$ and $100.0 \%$ (17/17) of the patients analyzed, respectively. The median survival duration of the patients who received allo-HSCT was 49.0 months, compared with 24.0 months for those who received chemotherapy, and the estimated 3 -year OS was $72.7 \%$ and $40.0 \%$, respectively, for patients who received allo-HSCT vs. those who received chemotherapy. When the patients that received allo HSCT and chemotherapy together were pooled, the estimated 3 year OS was $58.5 \%$.

\section{Discussion}

The formation of the SET-NUP214 fusion gene is caused by del(9)(q34.11q34.13) (3-5,8-10), or occasionally $\mathrm{t}(9 ; 9)(\mathrm{q} 34 ; \mathrm{q} 34)(1,5)$. The SET-NUP214 fusion gene has been previously reported in T-ALL, but rarely in AML and AUL (1-12). However, it has not been be reported thus far in B-ALL. In the present study, the first case of B-ALL carrying the SET-NUP214 fusion gene is reported, and a review of the literature is conducted.

Due to its rarity, the clinical characteristics and outcome of the SET-NUP214 rearrangement remain to be elucidated. To date, solely 42 cases carrying SET-NUP214, including the present case, have been reported. The majority of these patients experienced T-ALL (38/42; 90.4\%), while other subtypes of leukemia accounted for $<10 \%$, including AUL (4.8\%), AML $(2.4 \%)$ and B-ALL (2.4\%). Ben Abdelali et al (12) reported an incidence of SET-NUP214 of 5.6\% among 196 patients with T-ALL, which was slightly lower than that reported by previous studies in China and Korea concerning 59 (10.3\%) and $40(10.0 \%)$ patients with T-ALL, respectively $(8,10)$. According to previous studies, the incidence of SET-NUP214 among patients with T-ALL appears to be $\sim 3.0-10.3 \%(3,4,7,8,10,12)$.

In previous studies, the clinical presentation of patients with T-ALL carrying SET-NUP214 was not distinct from those not carrying SET-NUP214 (11). However, lymph node, spleen or liver enlargement and mediastinal involvement were frequently detected $(2,9)$. The median age of these patients was 27.5 years, and male patients accounted for $72.5 \%$ of all cases. Their median WBC count was $30.9 \times 10^{9}$ cells $/ 1$, and the median percentage of leukemic blasts in the bone marrow was remarkably high (82.0-97.0\%), which may reflect the high proliferation status of this rearrangement.

The most remarkable immunophenotype of the leukemic cells carrying the SET-NUP214 fusion gene was typically extreme immaturity, including expression of CD34, a specific marker for stem and progenitor cells, and CD7, a characteristic marker of immature T-cells, in 81.5 and $100.0 \%$ of patients, respectively (9). Additionally, myeloid markers such as CD33 and CD13, the expression of which has been reported in 


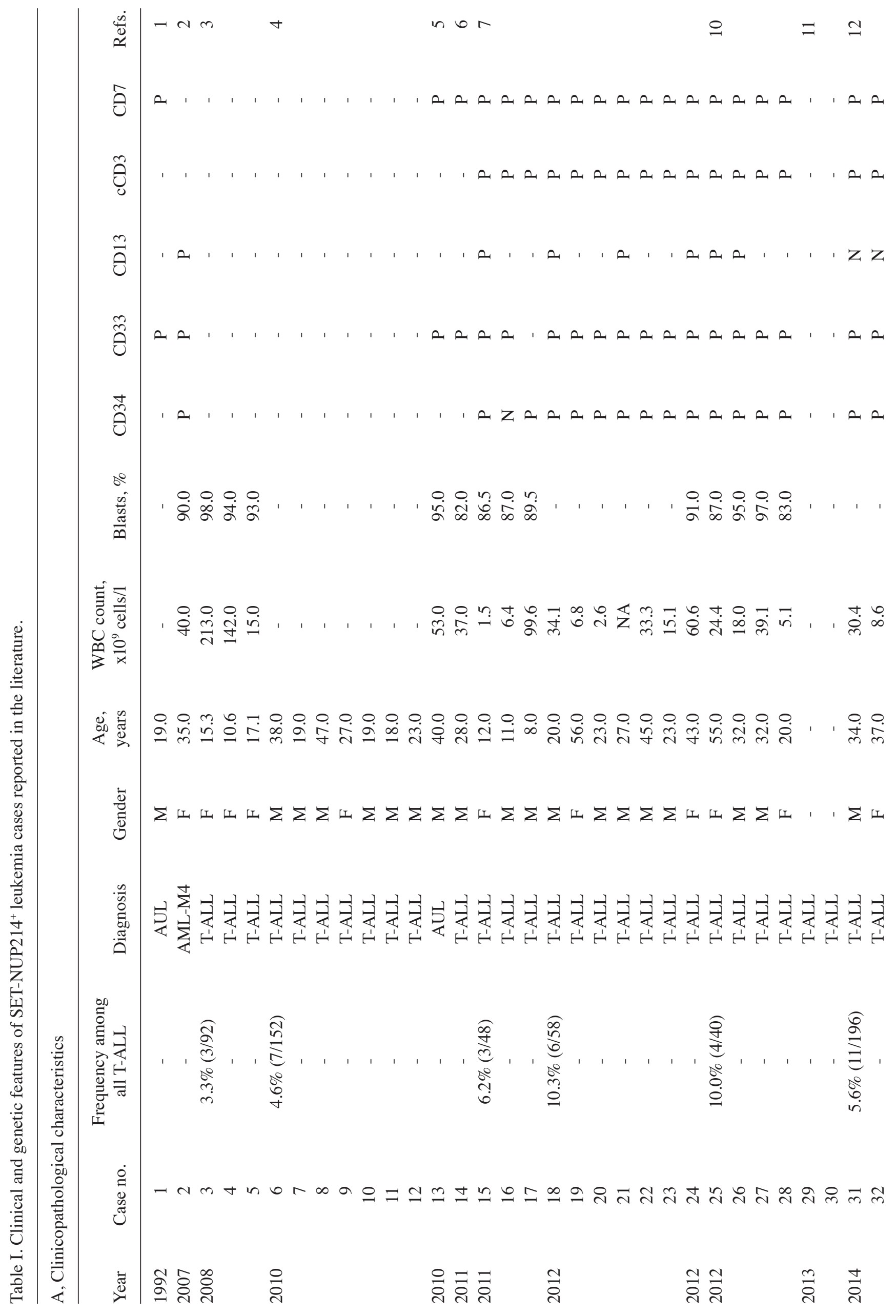




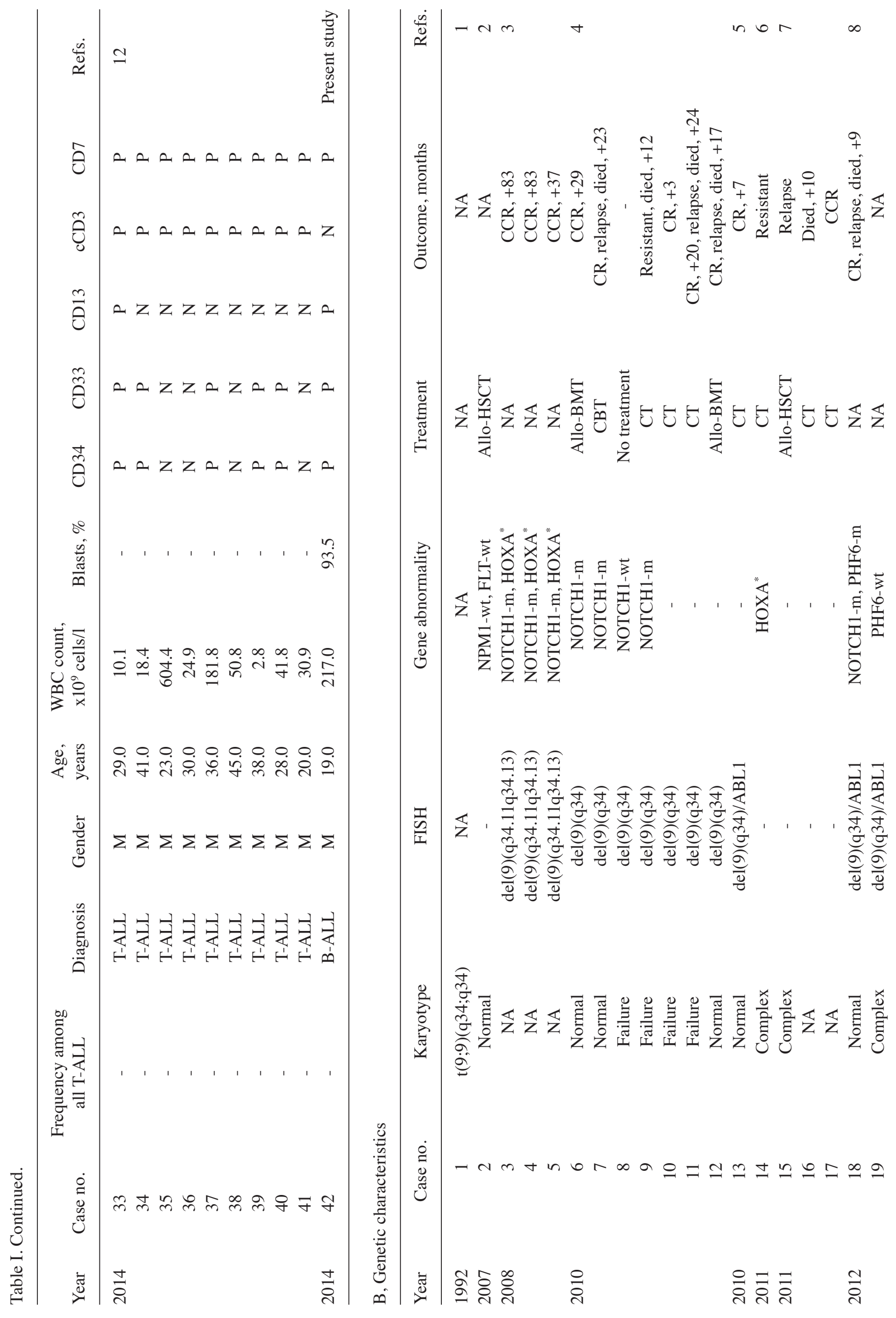




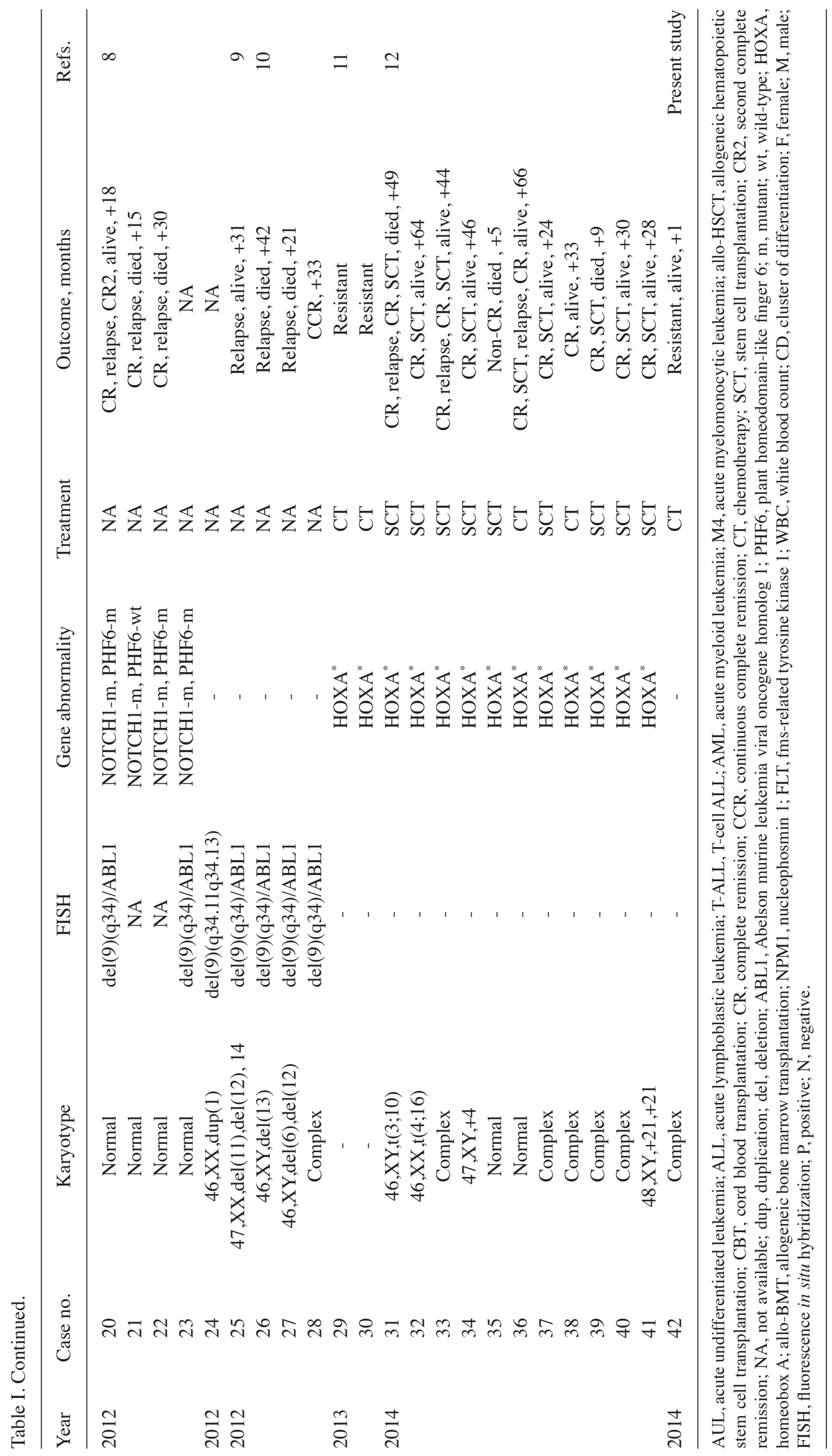


$19.0 \%(43 / 227)$ of T-ALL cases (13), were highly expressed in $86.2 \%$ and $47.4 \%$ of patients with SET-NUP214, respectively (13). The consistent expression of myeloid markers in the SET-NUP214 T-cell blasts suggests that malignant transformation may have occurred in cells arrested at early stages of myeloid or $\mathrm{T}$ lymphoid differentiation (10). The patient of the present case, despite presenting B-ALL, also exhibited a common T-cell immunophenotype, including CD $34^{+}, \mathrm{CD}_{3}{ }^{+}$, $\mathrm{CD}_{13}{ }^{+}$and $\mathrm{CD}^{+}$. The reason why the SET-NUP214 rearrangement typically induces the expression of myeloid lineage markers such as CD33 and CD13 remains unknown, and must be explored in future studies.

It has been previously reported that cryptic $\operatorname{del}(9)(q 34.11 q 34.13)$ and $t(9 ; 9)(q 34 ; q 34)$ are difficult to detect by analysis of the chromosomal karyotype using conventional G-banding (4). According to the literature, only 1 out of 31 patients was identified to carry $\mathrm{t}(9 ; 9)(\mathrm{q} 34 ; \mathrm{q} 34)$ using the above method (1-12). By contrast, FISH analysis detected the majority of patients carrying SET-NUP214 (3-5,8-10,12). Using a commercially available Abelson murine leukemia viral oncogene homolog 1 (ABL1) probe, all the SET-NUP214 cases associated with del(9)(q34) were identified (4). Therefore, the use of ABL1 FISH for patients with T-ALL is recommended, due to the cryptic nature of this rearrangement, followed by confirmation via multiplex RT-PCR to identify the SET-NUP214 fusion gene. The latter method has enabled the identification of a number of patients with the SET-NUP214 fusion gene $(7,9,11,14)$.

To date, the detailed mechanism by which SET-NUP214 mediates leukemogenesis has not been fully elucidated. Ozbek et al (15) observed expansion of an early progenitor cell pool and partial depletion of lymphocytes in SET-NUP214-carrying mice, although these animals were not prone to leukemia and did not exhibit shortening of disease latency following retroviral tagging. These results suggest that the SET-NUP214 fusion gene may determine the primitive phenotype of the disease, while secondary genetic lesions may be required for the development of the disease (15). SET, also referred to as TATA box binding protein-associated factor 1, was reported to be a putative oncogene that participates in transcription by modulating the organization of chromatin (16). SET is a component of inhibitor of histone acetyltransferase (INHAT), which participates in transcriptional activation (17). The NUP214 gene, also referred to as CAN, maps to chromosome $9 q 34$, and codes for a NUP containing phenylalanine-glycine repeats that resides in the cytoplasmic face of the nuclear pore complex and is implicated in nucleocytoplasmic transport, including the import and export of messenger RNA (18). NUP214 participates in development and possibly in leukemogenesis $(18,19)$. Overexpression of the HOXA gene has been proposed to be crucial for leukemic transformation (3). In addition, mutations in the PHF6 and NOTCH1 genes are frequently observed in patients with T-ALL carrying SET-NUP214, which may represent potential secondary genetic lesions of the leukemogenic event $(3,4,8,9,20)$.

Previous studies have indicated a poor treatment response and prognosis among patients carrying the SET-NUP214 fusion gene who were treated with chemotherapy $(4,5,8,11)$. SET-NUP $214^{+}$patients exhibited marked resistance to corticosteroids and chemotherapy in response to induction therapy (11), which may be due to a combination of various concomitant molecular events and complex genetic aberrations. In the case of the present report, the proportion of lymphoblasts following one week of conventional CODP chemotherapy was $96.0 \%$ of blood cells and $97.5 \%$ of bone marrow cells, and non-response was detected subsequently to the treatment, which is in agreement with the corticosteroid and chemotherapy resistance previously reported in patients with SET-NUP214 (12), and indicated poor prognosis. Of all the SET-NUP214 cases reported to date, clear outcome information was only available for 36 patients. The median survival time of the patients was 49.0 months, and the estimated 3 -year OS was $72.7 \%$. It has been previously reported that the outcome of SET-NUP214 ${ }^{+}$patients was similar to that of SET-NUP214- patients following allo-HSCT, suggesting that the latter is the most suitable treatment strategy for patients carrying SET-NUP214. The patient of the present case report is currently undergoing a second course of induction therapy and awaiting for allo-HSCT.

In conclusion, the present report demonstrates that SET-NUP214 is a recurrent oncogenic fusion gene associated with certain high risk factors and poor treatment response in adult patients. Due to the limited number of cases of SET-NUP214 B-ALL, an improved understanding of SET-NUP214 rearrangement, including its frequency, prognostic significance and certain clinical characteristics, would aid to define a novel specific subtype of acute leukemia and guide its treatment, since at present, HSCT is the best available treatment strategy for SET-NUP214+ patients with ALL.

\section{Acknowledgements}

The present study was supported by grants from the National Natural Science Foundation of China (Beijing, China; grant nos. 81370639 and 81170483) and the Beijing Municipal Science and Technology Commission (Beijing, China; grant no. Z141107002514004).

\section{References}

1. von Lindern M, Breems D, van Baal S, Adriaansen $H$ and Grosveld G: Characterization of the translocation breakpoint sequences of two DEK-CAN fusion genes present in $\mathrm{t}(6 ; 9)$ acute myeloid leukemia and a SET-CAN fusion gene found in a case of acute undifferentiated leukemia. Genes Chromosomes Cancer 5: 227-234, 1992.

2. Rosati R, La Starza R, Barba G, Gorello P, Pierini V, Matteucci C, Roti G, Crescenzi B, Aloisi T, Aversa F, et al: Cryptic chromosome $9 \mathrm{q} 34$ deletion generates TAF-Ialpha/CAN and TAF-Ibeta/CAN fusion transcripts in acute myeloid leukemia. Haematologica 92: 232-235, 2007.

3. Van Vlierberghe P, van Grotel M, Tchinda J, Lee C, Beverloo HB van der Spek PJ, Stubbs A, Cools J, Nagata K, Fornerod M, et al: The recurrent SET-NUP214 fusion as a new HOXA activation mechanism in pediatric T-cell acute lymphoblastic leukemia. Blood 111: 4668-4680, 2008

4. Gorello P, La Starza R, Varasano E, Chiaretti S, Elia L, Pierini V, Barba G, Brandimarte L, Crescenzi B, Vitale A, et al: Combined interphase fluorescence in situ hybridization elucidates the genetic heterogeneity of T-cell acute lymphoblastic leukemia in adults. Haematologica 95: 79-86, 2010.

5. Kim J, Lee SG, Song J, Kim SJ, Rha SY, Lee KA, Park TS and Choi JR: Molecular characterization of alternative SET-NUP214 fusion transcripts in a case of acute undifferentiated leukemia. Cancer Genet Cytogenet 201: 73-80, 2010. 
6. Lee SG, Park TS, Cho SY, Lim G, Park GJ, Oh SH, Cho EH, Chong SY and Huh JY: T-cell acute lymphoblastic leukemia associated with complex karyotype and SET-NUP214 rearrangement: A case study and review of the literature. Ann Clin Lab Sci 41: 267-272, 2011.

7. Li WJ, Cui L, Gao C, Zhao XX, Liu SG, Xin YP, Zhang RD, Zhang DW, Wang B, Li ZG and Wu MY: Gene rearrangement pattern of immunoglobulin and T-cell receptor (Ig/TR) and its clinical characteristics in children with SET-NUP214 fusion gene-positive leukemia/lymphoma. Zhongguo Shi Yan Xue Ye Xue Za Zhi 19: 1362-1367, 2011 (In Chinese).

8. Dai HP, Wang Q, Wu LL, Ping NN, Wu CX, Xie JD, Pan JL, Xue YQ, Wu DP and Chen SN: Expression of SET-NUP214 fusion gene in patients with T-cell acute lymphoblastic leukemia and its clinical significance. Zhongguo Shi Yan Xue Ye Xue Za Zhi 20: 1047-1051, 2012 (In Chinese).

9. Lee EY, Park TS, Kim MJ, Chang MH, Cho EH, Park SJ, Choi JR and Yoo JH: Detection of SET-NUP214 rearrangement using multiplex reverse transcriptase-polymerase chain reaction (RT-PCR) in acute leukemias: A case report and literature review on a Korean case series. Ann Hematol 91: 1135-1138, 2012.

10. Chae H, Lim J, Kim M, Park J, Kim Y, Han K, Lee S and Min WS: Phenotypic and genetic characterization of adult T-cell acute lymphoblastic leukemia with del(9)(q34); SET-NUP214 rearrangement. Ann Hematol 91: 193-201, 2012.

11. Liu F, Gao L, Jing Y, Xu YY, Ding Y, Zhou MH, Ma C, Li MY, Sun JZ, Wang LL and Yu L: Detection and clinical significance of gene rearrangements in Chinese patients with adult acute lymphoblastic leukemia. Leuk Lymphoma 54: 1521-1526, 2013.

12. Ben Abdelali R, Roggy A, Leguay T, Cieslak A, Renneville A, Touzart A, Banos A, Randriamalala E, Caillot D, Lioure B, et al: SET-NUP214 is a recurrent $\gamma \delta$ lineage-specific fusion transcript associated with corticosteroid/chemotherapy resistance in adult T-ALL. Blood 123: 1860-1863, 2014.
13. Uckun FM, Sather HN, Gaynon PS, Arthur DC, Trigg ME, Tubergen DG, Nachman J, Steinherz PG, Sensel MG and Reaman GH: Clinical features and treatment outcome of children with myeloid antigen positive acute lymphoblastic leukemia: A report from the Children's Cancer Group. Blood 90: 28-35, 1997.

14. Kim MJ, Choi JR, Suh JT, Lee HJ, Lee WI and Park TS: Diagnostic standardization of leukemia fusion gene detection system using multiplex reverse transcriptase-polymerase chain reaction in Korea. J Korean Med Sci 26: 1399-1400, 2011.

15. Ozbek U, Kandilci A, van Baal S, Bonten J, Boyd K, Franken P, Fodde R and Grosveld GC: SET-CAN, the product of the $\mathrm{t}(9 ; 9)$ in acute undifferentiated leukemia, causes expansion of early hematopoietic progenitors and hyperproliferation of stomach mucosa in transgenic mice. Am J Pathol 171: 654-666, 2007.

16. Seo SB, McNamara P, Heo S, Turner A, Lane WS and Chakravarti D: Regulation of histone acetylation and transcription by INHAT, a human cellular complex containing the set oncoprotein. Cell 104: 119-130, 2001.

17. Muto S, Senda M, Akai Y, Sato L, Suzuki T, Nagai R, Senda T and Horikoshi M: Relationship between the structure of SET/TAF-Ibeta/INHAT and its histone chaperone activity. Proc Natl Acad Sci USA 104: 4285-4290, 2007.

18. $\mathrm{Xu} \mathrm{S}$ and Powers MA: Nuclear pore proteins and cancer. Semin Cell Dev Biol 20: 620-630, 2009.

19. van Deursen J, Boer J, Kasper L and Grosveld G: G2 arrest and impaired nucleocytoplasmic transport in mouse embryos lacking the proto-oncogene CAN/Nup214. EMBO J 15: 5574-5583, 1996.

20. Wang Q, Qiu H, Jiang H, Wu L, Dong S, Pan J, Wang W, Ping N, Xia J, Sun A, et al: Mutations of PHF6 are associated with mutations of NOTCH1, JAK1 and rearrangement of SET-NUP214 in T-cell acute lymphoblastic leukemia. Haematologica 96: 1808-1814, 2011. 